\title{
CARACTERIZAÇÃO DAS CINZAS DO BAGAÇO DE CANÁ-DE-AÇÚCAR COMO MATERIAL ALTERNATIVO PARA A REDUÇÃO DE IMPACTOS AMBIENTAIS
}

\section{Tainara Rigotti de Castro, MSc. (UNESPAR); Carlos Humberto Martins, Dr. (UEM).} PALAVRAS CHAVE

Cinza do bagaço de cana-de-açúcar; Material alternativo; Impactos ambientais.

KEY WORDS

Sugarcane bagasse ash; Alternative Material; Environmental impacts

\section{RESUMO}

Devido à necessidade de diminuir a utilização de áreas para a deposição de cinzas, bem como a necessidade da diminuição da utilização de materiais na construção civil e impactos ambientais causados por sua extração, esta pesquisa tem por objetivo realizar a caracterização da cinza pesada e da cinza volante do bagaço de cana-de-açúcar, a fim de apontar se tais amostras possuem as características necessárias para substituir a areia e o cimento, respectivamente. Para tal, as cinzas foram caracterizadas conforme procedimentos metodológicos da Associação Brasileira de Normas Técnicas (ABNT), quanto à distribuição granulométrica; índice de pozolanicidade; massa específica; teor de umidade; perda ao fogo; composição química, e; contaminantes. A cinza pesada se mostrou um material com características semelhantes às da areia, podendo ser fonte viável de adição mineral em matrizes cimentícias; e a cinza volante comprovou sua reatividade e a possibilidade de substituir parcialmente o cimento.

\footnotetext{
ABSTRACT

Front of the need to reduce the use of ash disposal areas, as well as the need to decrease the use of construction materials and environmental impacts caused by its extraction, this research aims to carry out the characterization of sugar cane bagasse ashes, in order to point if such samples have the features needed to replace the cement and sand. The ashes were characterized as methodological procedures of the Associação Brasileira de Normas Técnicas (ABNT), as the particle size distribution, pozzolanic activity, specific mass, fire loss, chemical composition and contaminants. The heavy ash proved is material with similar features to the sand, and can be a viable source of mineral addition in cements; and the fly ash proved have pozzolanic reactivity and the possibility to partially replace the cement.
} 


\section{INTRODUÇÃO}

Impulsionada pela urbanização e industrialização, a preocupação com a saúde ambiental se iniciou em 1970, quando se intensificaram os danos gerados pelos excessos de impactos causados pela produção em escala industrial (MAURY; BLUMENSCHEIN, 2012). O setor da construção civil chama atenção neste ponto, apresentando fatores negativos, tais como: processos produtivos ineficientes e ultrapassados; geração de resíduos, e; consumo desenfreado e impensado de materiais, gerando uma série de impactos ambientais.

Esse setor é essencial para o crescimento de uma nação, desempenhando um papel importante e vital para sociedade, transformando necessidades em infra-estrutura (IBRAHIM et al., 2010). Entretanto, o atendimento dessas necessidades vem acompanhado de extrações de matérias-primas, seguido da degradação do meio ambiente (BROWN; LUGO, 1994).

O cimento e a areia são utilizados no setor da construção civil, sendo retirados do solo através da operação de mineração, causando impactos ambientais. A melhor maneira de diminuir a utilização desses recursos e conservar o meio ambiente é o emprego de formas alternativas, como por exemplo, o uso de resíduos industriais como matéria-prima (ALWAELI, 2013). Assim, além da redução da demanda da extração de recursos naturais ainda há a possibilidade de obtenção de produtos com propriedades superiores.

Neste âmbito, a grande quantidade de resíduos industriais produzidos em todo o mundo implica que atualmente, a sua reciclagem seja extremamente necessária, não só devido ao aumento do custo de sua deposição em aterros, que por sua vez, se reflete no custo dos produtos, mas também como uma consequência do desperdício zero, que deve ser o objetivo final de todas as futuras atividades humanas (FARAONE et al., 2009).

A incorporação de resíduos no emprego de formas alternativas tem mostrado resultados satisfatórios na literatura, principalmente na utilização de cinzas do bagaço de cana-de-açúcar, no ramo da construção civil (GONZÁLEZ-LÓPEZ et al., 2015; CHEN et al., 2006; LIMA et al., 2012; SOUZA et al., 2011; AKRAM; MEMON; OBAID, 2009; CORDEIRO et al., 2009), através de adições e substituições parciais de agregados e aglomerantes, tanto em concretos, pastas de cimento e argamassas, quanto em materiais cerâmicos e blocos de solo.

Devido à necessidade de diminuir a utilização de áreas para a deposição de cinzas, bem como a necessida- de da diminuição da utilização de materiais na construção civil e impactos ambientais causados por sua extração, esta pesquisa tem por objetivo realizar a caracterização da cinza volante e da cinza pesada do bagaço de cana-de -açúcar, a fim de apontar se tais amostras possuem as características necessárias para substituir o cimento e a areia, respectivamente.

\section{REVISÃO TEÓRICA}

\subsection{Impactos e degradação ambiental}

Uma das principais atividades que acarretam impactos para o meio ambiente é a mineração. A extração pode ser realizada através de vários métodos e seu objetivo é a retirada de substâncias a partir de depósitos ou massas minerais. No ano de 2011, o Brasil registrou o número de 8.870 mineradoras presentes em seu território (IBRAM, 2012).

Com a prática desta atividade todos os componentes ambientais tornam-se influenciados, sendo denunciada por uma ampla manifestação visual, trazendo mudanças drásticas para a paisagem, cobrindo frequentemente uma área tão vasta que é impossível restaurá-la ao seu estado original novamente (DULIAS, 2010).

Os principais impactos causados ao meio ambiente pela extração de minérios são subsidência, contaminação e escassez das águas, destruição de habitat, extinção e escassez de fontes e jazidas, alterações na flora e fauna do entorno destes locais de exploração, reconfiguração das superfícies topográficas, aceleração do processo erosivo, modificações de cursos d'água, interceptação do lençol freático, aumento da emissão de gases e partículas em suspensão no ar, aumento de ruídos e propagações de vibrações, resultando em áreas degradadas (ROTH; GARCIAS, 2009).

Além disso, essas áreas não possuem mais a capacidade de repor as perdas de matéria orgânica do solo, nutrientes, biomassa e estoque de propágulos, alterando as características biológicas, físicas e químicas do local explorado, tornando o solo estéril (BROW; LUGO, 1994).

A extração de calcário, para a produção de cimento; e a extração de areia, largamente utilizado como materiais na construção civil; são realizadas através da mineração.

O cimento, por sua vez, além da necessidade da retirada das pedras de calcário do solo, necessita da operação de calcinação, dada através da queima de combustíveis necessários para manter as altas temperaturas necessárias nos fornos utilizados (aproximadamente $1500^{\circ} \mathrm{C}$ ). 
Sendo assim, a indústria do cimento além de degradar áreas, ainda é considerada uma das principais contribuintes para as emissões dos gases de efeito estufa (ALI, SAIDUR, HOSSAIN, 2011).

O mercado cimenteiro, no Brasil, é composto tanto por grupos nacionais, quanto estrangeiros, com 81 fábricas espalhadas por todas as regiões brasileiras e com uma capacidade instalada anunciada de 78 milhões de toneladas/ano (CIMENTO.ORG, 2012). Desta forma, o Brasil está entre os 10 maiores produtores de cimento no mundo.

A areia é um material de granulometria variada, composto essencialmente de sílica e que passou por um processo de beneficiamento, suas fontes de produção são representadas por depósitos de areia quartzosa, arenito ou quartzito (FERREIRA; DAITX, 2000). De acordo com o tipo de depósito mineral, varia o processo de retirada do solo (lavra), que geralmente é por dragagem ou desmonte hidráulico. O mercado brasileiro é atendido por uma ampla e diversificada gama de produtores, envolvendo cerca de 2500 empresas de extração de areia (ANEPAC, 2013a).

Neste contexto, a redução da utilização tanto do cimento, como da areia é considerada importante para combater os problemas de degradação e poluição do meio ambiente.

\subsection{Cinzas do bagaço da cana-de-açúcar}

No processo de beneficiamento da cana-de-açúcar o maior subproduto gerado é o bagaço da cana-de-açúcar, utilizado como combustível em caldeiras para geração de energia que resulta em cinzas. Se for levado em consideração que na safra 2013/2014 a colheita de 652 milhões de toneladas de cana-de-açúcar, e que todo o bagaço foi utilizado como fonte de energia, foram então geradas aproximadamente 3,9 milhões de toneladas de cinza (CONAB, 2013).

Parte da cinza gerada, dita cinza pesada, é depositada num cinzeiro abaixo da grelha da caldeira e o restante, de distribuição granulométrica mais fina, dita cinza volante ou leve, é suspensa pelos gases de combustão e, antes de ser dispersa na atmosfera através da chaminé passa por algum processo de limpeza de gases para redução nas emissões de particulados. Salienta-se que o processo de separação dessas cinzas difere de acordo com a tecnologia utilizada pela indústria.

Parte dessas cinzas volta para o solo dos canaviais para ser utilizada como adubo, apesar de ser um componente pobre em nutrientes e de difícil deterioração, ainda possui em sua composição metais pesados, podendo con- taminar o solo e lençóis freáticos. A literatura ressalta que esta prática é comum entre os agricultores de cana, chamada de iniciativa ambientalmente correta, eliminada juntamente à torta de filtro e vinhaça, porém, ignora o uso de agrotóxicos nas plantações, fazendo com que estes produtos persistam no solo juntamente com as cinzas (SALES; LIMA, 2010). As cinzas, quando descartadas de maneira inadequada, podem causar a contaminação dos solos adjacentes, águas subterrâneas e problemas de saúde, ocasionando consequentemente graves problemas sociais e ambientais. Dessa forma, não possuindo outro modo de utilização, o correto é que esse resíduo seja destinado aos aterros sanitários (FRÍAS; VILLAR; SAVASTANO, 2011).

Andrade et al. (2007) ressalta que as cinzas pesadas são capazes de substituir a areia natural, tendo potencial de aproveitamento para produção de materiais à base de cimento. Cordeiro et al. (2008) afirma que a cinza volante do bagaço de cana-de-açúcar tem potencial para ser utilizada como adição mineral, substituindo parte do cimento em argamassas e concretos.

Neste contexto, o encaminhamento desse resíduo de forma adequada é considerado importante para combater os problemas de poluição do meio ambiente.

\section{METODOLOGIA}

Os materiais foram coletadas em uma Usina localizada no Noroeste do Paraná/PR. A cinza pesada foi coletada em sacos plásticos e preparada em laboratório, para posterior caracterização. Antes dos procedimentos ela foi peneirada em malha 0,6 para a retirada de folhas e pedaços de bagaço com queima incompleta. $\mathrm{O}$ aspecto da cinza pesada pode ser visualizado através da Figura $1 a$. A cinza volante foi coletada em sacos plásticos e tratada em laboratório para posterior caracterização. Antes dos procedimentos ela foi seca em temperatura ambiente por $48 \mathrm{~h}$ e após seca em estufa por mais $48 \mathrm{~h}$ para a retirada de umidade. Após a etapa de secagem, o material foi desgrumado com o auxílio de um almofariz. O aspecto da cinza leve pode ser visualizado na Figura 1b. 
Figura 1 - (a) Aspecto da cinza leve; (b) Aspecto da cinza pesada. Fonte: Elaborado pelos autores.
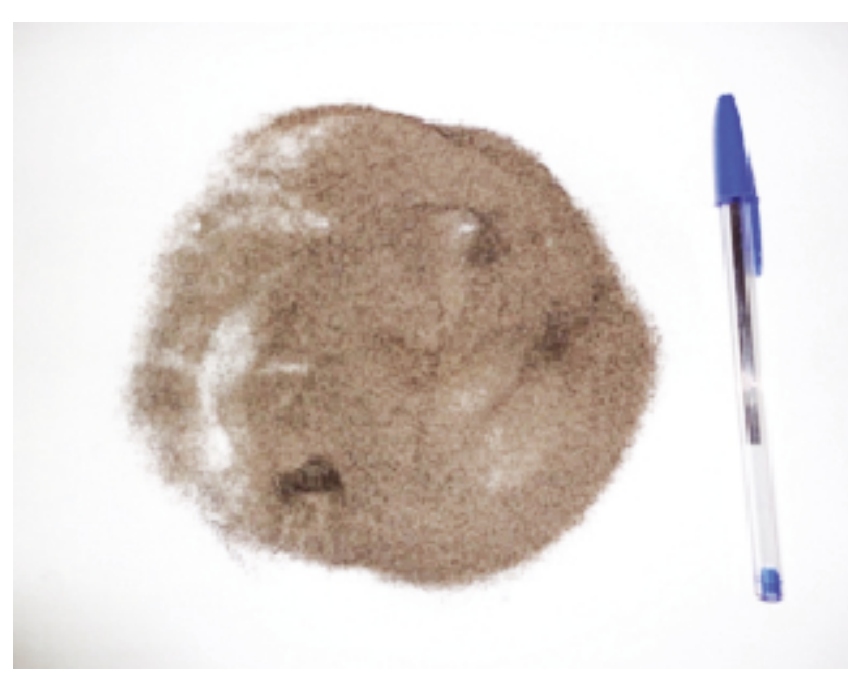

Quanto aos métodos, a distribuição das partículas das amostras de cinzas foi determinada pela combinação dos procedimentos de peneiramento e sedimentação conforme sugere a NBR 7181 (ABNT, 1984b). A atividade pozolânica foi determinada através do Método de Chapelle modificado (ABNT, 2010). Através da ABNT NBR 6508 (ABNT, 1984a), foi determinada a massa específica de cada amostra de cinza. O teor de umidade foi determinado a partir da secagem das amostras de resíduos em estufa por 24h, conforme especificações da NBR NM 24 (ABNT, 2003), e o teor de perda ao fogo foi determinado conforme a NBR NM 18 (ABNT, 2012), a partir da calcinação das amostras em

\section{RESULTADOS E DISCUSSÕES}

As curvas de distribuição de tamanho de partículas da cinza pesada deixam claro que esta possui partículas de maior diâmetro, quando comparadas às da cinza volante (Figura 2). Os resultados revelaram que $51 \%$ da amostra da cinza pesada ficou retida nas peneiras de 0,06 a 0,2 mm, e de acordo com a classificação da NBR 6502 (ABNT, 1995) são semelhantes à areia fina. A cinza volante teve $81 \%$ da amostra retida, sendo semelhante ao silte. As amostras das cinzas indicaram pouca variação do diâmetro das partículas, o que indicou uma amostra uniforme, com distribuição das partículas bem graduada.

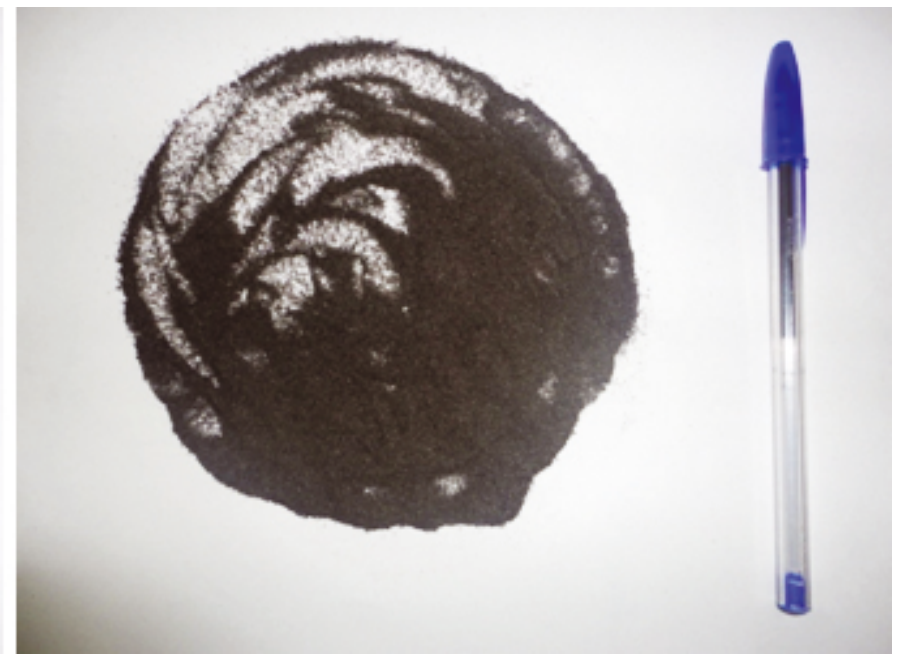

Fonte: Elaborado pelos autores.

forno mufla à temperatura entre $950 \pm 50{ }^{\circ} \mathrm{C}$ por no mínimo 50 min. A composição química das amostras foi determinada utilizando espectrômetro de raios-X Rigaku, com radiação $\mathrm{Pd} \mathrm{Ka}$, corrente de 1,2 mA e voltagem de $40 \mathrm{kV}$. Os contaminantes presentes nas cinzas foram analisados por meio da obtenção do extrato lixiviado, seguindo especificações da NBR 10005 (ABNT, 2004b) e solubilizado, de acordo com a NBR 10006 (ABNT, 2004c) para determinação dos teores de contaminantes de acordo constantes na NBR 10004 (ABNT, 2004a), por meio de Espectrômetro de Absorção Atômica (EAA 52 Varian Spectraa - 240FS) e Cromatôgrafo de íons (Metrohm - 850 Professional IC). 
Figura 2: Curvas de distribuição granulométrica das cinzas do bagaço de cana-de-açúcar.

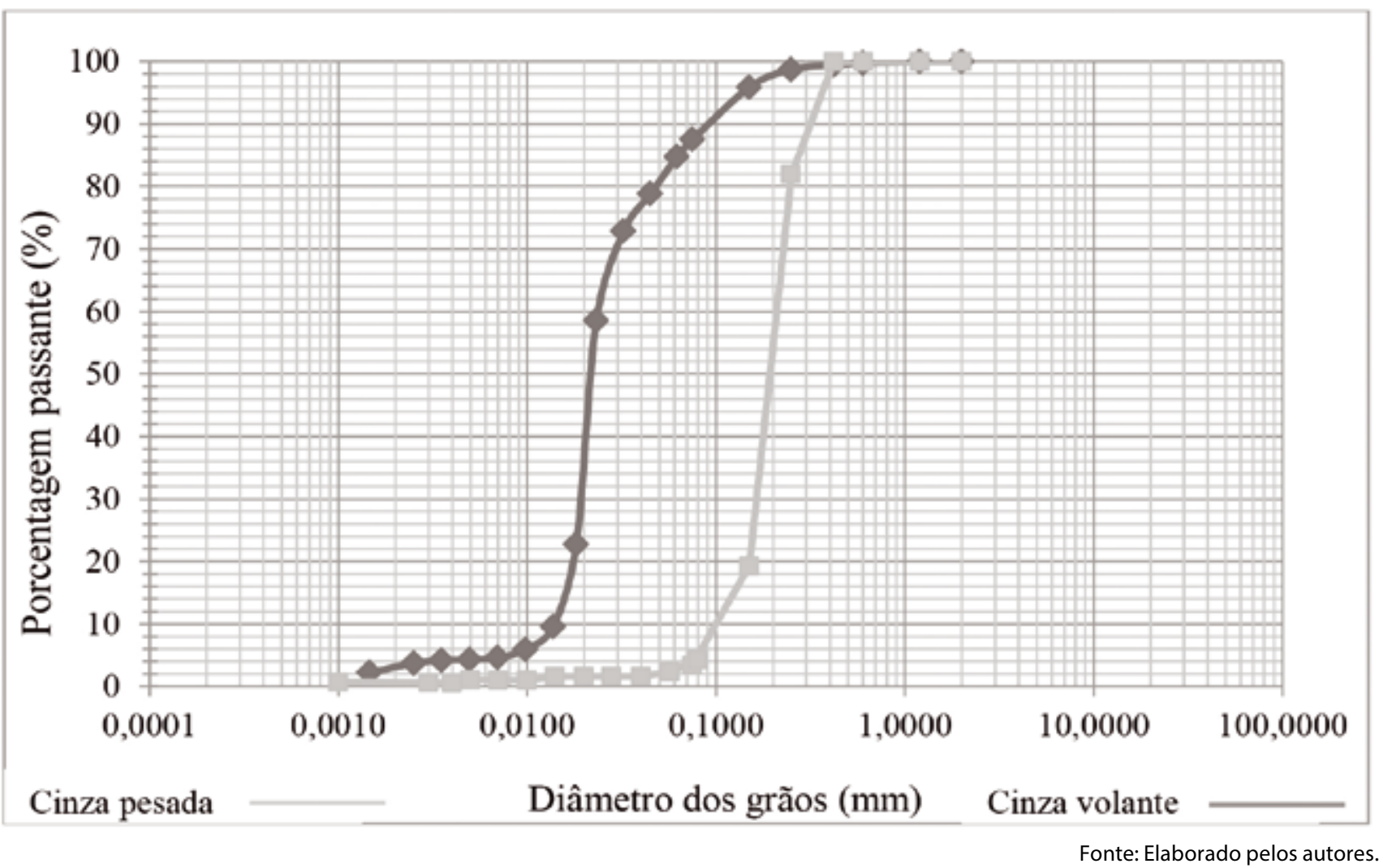

Um material para ser pozolânico, de acordo com a NBR 15895 (ABNT, 2010), deve apresentar uma pozalinicidade acima de $436 \mathrm{mg} \mathrm{Ca}(\mathrm{OH}) 2 /$ pozolana. $\mathrm{O}$ valor obtido através da amostra de cinza pesada resultou em $101 \mathrm{mg}$ $\mathrm{Ca}(\mathrm{OH}) 2 / \mathrm{g}$ amostra, o que caracteriza que esta cinza não possui atividade pozolânica. $\mathrm{O}$ valor obtido para a cinza volante foi de $569 \mathrm{mg} \mathrm{Ca}(\mathrm{OH}) 2 / \mathrm{g}$ amostra, o que caracteriza que pode ser viável sua substituição por cimento.

Em relação a massa específica, se encontrou um valor de $2,64 \mathrm{~g} / \mathrm{cm} 3$ para cinza pesada e de $2,75 \mathrm{~g} / \mathrm{cm} 3$ para cinza volante. Em comparação com a massa específica da areia e do cimento, respectivamente, valores semelhantes são observados: 2,65 g/cm3 (SALES; LIMA, 2010) e 2,75 g/ cm3 (VOTORANTIM, 2014).

Através da determinação do teor de umidade se pode constatar o quão a cinza leve é mais úmida $(1,68 \%)$, em relação à cinza pesada (0,16\%).

O teor de perda ao fogo da cinza pesada e da cinza volante foram de $13,92 \%$ e 9,56\%, respectivamente. Agredo et al. (2014), encontraram semelhantemente, em suas amostras de cinzas caracterizadas, valores próximos aos desta pesquisa, sendo de $14 \%$ para a cinza pesada e $10 \%$ para a cinza volante. Esta propriedade esta diretamente ligada à quantidade de matéria orgânica presente nestes resíduos.
As composições químicas das amostras de resíduos de cinzas são fornecidas pela Tabela 1. De acordo com os referidos dados, a cinza pesada contém uma grande quantidade de sílica $(57,41 \%)$, e menor grau de óxido de ferro $(21,79 \%)$. Para a cinza volante ocorre o inverso, uma maior presença de óxido de ferro $(47,98 \%)$ e um menor grau de sílica $(25,82 \%)$. Quando comparada a literatura as cinzas desta pesquisa tendem a ter uma menor quantidade de sílica, onde foram encontradas: cinza pesada com um teor de $66,61 \%$ de sílica (FRÍAS; VILLAR; SAVASTANO, 2011) e cinza volante com uma porcentagem de 61,59\% (FARIA; GURGEL; HOLANDA, 2012) e 47,4\% (UYGUNOGLU et al., 2012). Estes resultados podem estar relacionados com as diferenças de solos onde a cana é cultivada, métodos de fertilização do solo, métodos de queima do bagaço, entre outros. 
Tabela 1: Composição química das Cinzas do bagaço de cana-de-açúcar.

\begin{tabular}{|c|c|c|c|}
\hline \multirow[t]{2}{*}{ Elemento } & \multirow[t]{2}{*}{ Fórmula Composta } & \multicolumn{2}{|c|}{ Concentração (\%) } \\
\hline & & Cinza pesada & Cinza volante \\
\hline $\mathrm{Si}$ & $\mathrm{SiO}_{2}$ & 57,41 & 25,82 \\
\hline $\mathrm{Fe}$ & $\mathrm{Fe}_{2} \mathrm{O}_{3}$ & 21,79 & 47,98 \\
\hline $\mathrm{Ti}$ & $\mathrm{TiO}_{2}$ & 6,41 & 7,16 \\
\hline $\mathrm{Al}$ & $\mathrm{Al}_{2} \mathrm{O}_{3}$ & 4,31 & 10,57 \\
\hline K & $\mathrm{K}_{2} \mathrm{O}$ & 4,05 & 4,15 \\
\hline $\mathrm{Ca}$ & $\mathrm{CaO}$ & 1,96 & 1,53 \\
\hline $\mathrm{P}$ & $\mathrm{P}_{2} \mathrm{O}_{5}$ & 1,14 & 0,79 \\
\hline $\mathrm{Mg}$ & $\mathrm{MgO}$ & 1,03 & 0,68 \\
\hline V & $\mathrm{V}_{2} \mathrm{O}_{5}$ & 0,72 & - \\
\hline $\mathrm{Cl}$ & $\mathrm{Cl}$ & 0,46 & 0,19 \\
\hline $\mathrm{Mn}$ & $\mathrm{MnO}$ & 0,37 & 0,73 \\
\hline S & $\mathrm{SO}_{3}$ & 0,25 & - \\
\hline $\mathrm{Zr}$ & $\mathrm{ZrO}_{2}$ & 0,11 & 0,11 \\
\hline $\mathrm{Cu}$ & $\mathrm{CuO}$ & - & 0,16 \\
\hline $\mathrm{Zn}$ & $\mathrm{ZnO}$ & - & 0,13 \\
\hline
\end{tabular}

Fonte: Elaborado pelos autores.

A Tabela 2 mostra que o teste de solubilização indicou a presença de metais pesados nas amostras de cinzas. Os resultados do extrato de lixiviação ficaram dentro do limite permitido para o grupo de compostos orgânicos, de acordo com o Anexo F da NBR 10004 (ABNT, 2004a). O ensaio de solubilização revelou elementos químicos excedendo o nível permitido empregado pelo anexo $G$ da referida norma. Portanto, as amostras aqui foram

Tabela 2: Elementos químicos encontrados no extrato solubilizado de

Cinzas do bagaço de cana-de-açúcar excedentes aos limites padrão da norma brasileira

\begin{tabular}{llll}
\hline Componentes & Limites $(\mathrm{mg} / \mathrm{l})$ & Cinza pesada & Cinza volante \\
\hline Alumínio & 0,2 & 0,79 & 2,54 \\
Chumbo & 0,01 & 0,12 & 0,12 \\
Cádmio & 0,006 & 0,005 & 0,021 \\
Manganês & 0,1 & 0,02 & 0,29 \\
\hline
\end{tabular}




\section{CONSIDERAÇÕES FINAIS}

A construção de edificações consome até $75 \%$ dos recursos extraídos da natureza, com o agravante de que a maior parte destes não são renováveis. Os impactos são evidentes, visto que muitas dessas explorações são feitas de forma incorreta, tornando o solo impróprio para a plantação. Sem dúvidas, é impossível minerar sem causar impacto ambiental, seja ele de maior ou menor extensão. Sabe-se que é impossível a sociedade viver sem os materiais oriundos desta atividade, entretanto, deve-se ressaltar a necessidade de se desenvolver a consciência de que é necessário reparar e minimizar os danos causados nessas áreas de exploração.

Desta forma, um dos grandes desafios da construção civil é diminuir o desperdício de materiais, bem como utilizar formas alternativas para o fabrico destes, a fim da diminuição da extração dos recursos naturais. Conciliar a atividade da construção civil com as condições que conduzam a um desenvolvimento sustentável consciente e menos agressivo ao meio ambiente é essencial.

As cinzas do bagaço de cana-de-açúcar, cuja quantidade gerada aumentará significativamente nos próximos anos, em decorrência da ampliação do setor de produção de álcool combustível, se mostraram, através da caracterização realizada nesta pesquisa, como um material alternativo viável. A cinza pesada se mostrou um material com características semelhantes às da areia, visto a sua distribuição granulométrica; massa específica; inexistência de atividade pozolânica; alto teor de sílica, podendo ser fonte viável de adição mineral em matrizes cimentícias. O índice da atividade pozolânica da cinza volante pode ser atribuída à presença de sílica amorfa; ao reduzido tamanho de partículas, e; à reduzida perda ao fogo, comprovando sua reatividade e a possibilidade de substituir parcialmente o cimento.

\section{REFERÊNCIAS}

1. AGREDO, J. T.; GUTIÉRREZ, R. M. de; GIRALDO, C. E. E.; SALCEDO, L. O. G. Characterization of sugar cane bagasse ash as supplementary material for Portland cement.Ingeniería e Investigación, v.34, n.1, p.5-10, 2014.

2. AKRAM, T.; MEMON, S. A.; OBAID, H. Production of low cost self compacting concrete using bagasse ash. Construction and Building Materials, v.23, p.703-712, 2009.

3. ALI, M. B.; SAIDUR, R.; HOSSAIN, M. S. A review on emission analysis in cement industries. Renewable and Sustainable Energy Reviews, v. 15, p. 2252-2261, 2011.

4. ALWAELI, M. Application of granulated lead-zinc slag in concrete as an opportunity to save natural resources. Radiation Physics and Chemistry, v.83, p.54-60, 2013.

5. ANDRADE, L. B.; ROCHA, J. C.; CHERIAF, M. Evaluation of concrete incorporating bottom ash as natural aggregates replacement. Waste Management, v.27, p.1190-99, 2007.

6. ASSOCIAÇÃO BRASILEIRA DE NORMAS TÉCNICAS. NBR 6502: Rochas e Solo. Rio de Janeiro, 1995.

7. ASSOCIAÇÃO BRASILEIRA DE NORMAS TÉCNICAS. NBR 6508. Massa específica dos Sólidos. Rio de Janeiro, 1984a.

8. ASSOCIAÇÃO BRASILEIRA DE NORMAS TÉCNICAS. NBR 7181. Solo - análise granulométrica. Rio de Janeiro, $1984 b$.

9. ASSOCIAÇÃO BRASILEIRA DE NORMAS TÉCNICAS. NBR 10004. Resíduos Sólidos - Classificação. Rio de Janeiro, 2004a.

10. ASSOCIAÇÃO BRASILEIRA DE NORMAS TÉCNICAS. NBR 10005. Procedimento para obtenção de extrato lixiviado de resíduos sólidos. Rio de Janeiro, 2004b.

11. ASSOCIAÇÃO BRASILEIRA DE NORMAS TÉCNICAS. NBR 10006. Procedimento para obtenção de extrato solubilizado de resíduos sólidos Rio de Janeiro, 2004c. ASSOSSIAÇÃO BRASILEIRA DE NORMAS TÉCNICAS. 
NBR 15895. Materiais pozolânicos - Determinação do teor de hidróxido de cálcio fixado - Método Chapelle modificado. Rio de Janeiro, 2010.

\section{ASSOCIAÇÃO BRASILEIRA DE NORMAS TÉCNICAS. NBR} NM 24. Materiais pozolânicos - Determinação do teor de umidade. Rio de Janeiro, 2003.

\section{ASSOCIAÇÃO BRASILEIRA DE NORMAS TÉCNICAS. NBR} NM 18. Cimento Portland - Análise química - Determinação de perda ao fogo. Rio de Janeiro, 2012.

14. ASSOCIAÇÃO NACIONAL DAS ENTIDADES DE PRODUTORES DE AGREGADOS PARA CONSTRUÇÃO CIVIL - ANEPAC. Agregados. 2013. Disponível em: <http://anepac.org. br/wp/agregados/>. Acesso em 8 de dezembro de 2015.

CIMENTO.ORG. Cimento Brasil. 2012. Disponível em: $<$ http://www.cimento.org/index.php?option=com_content $\&$ view=article\&id=46\&ltemid=18>. Acesso em 29 de novembro de 2013.

15. CHEN, C.; SUN, C.; GAU, S.; WU, C.; CHEN, Y. The effects of the mechanical-chemical stabilization process for municipal solid waste incinerator fly ash on the chemical reactions in cement paste. Waste Management, v.33, p.858-865, 2013.

16. CONAB - COMPANHIA NACIONAL DE ABASTECIMENTO. Acompanhamento da safra brasileira de Cana-de-Açúcar 2013/2014 - Segundo Levantamento.2013. Disponível em: http://www.conab.gov.br/OlalaCMS/uploads/arquivos/13_08_08_09_39_29_boletim_cana_portugues_ -abril_2013_1o_lev.pdf. Acesso em 16 de fevereiro de 2015. BROWN, S.; LUGO, A. E. Rehabilitation of tropical lands: a key to sustaining developing. Restoration Ecology, v. 2, p. 97-111, 1994.

17. CORDEIRO, G. C.; TOLEDO FILHO, R. D.; FAIRBAIRN, E. M. R.; TAVARES, L. M. M. Pozzolanic activity and filler effect of sugar cane bagasse ash in Portland cement and lime mortars. Cement \& Concrete Composites, v.30, p.410-418, 2008.

18. CORDEIRO, G. C.; TOLEDO FILHO, R. D.; TAVARES, L. M.; FAIRBAIRN, E. de M. R. Ultrafine grinding of sugar cane bagasse ash for application as pozzolanic admixture in concrete. Cement and Concrete Research, v.39, p.110-115, 2009.
DULIAS, R. Landscape planning in areas of sand extraction in the Silesian Upland, Poland. Landscape and Urban Planning, v. 95, p.91-104, 2010.

19. FARAONE, N., TONELLO, G., FURLANI, E., MASCHIO, S. Steelmaking slag as aggregate for mortars: effects of particle dimension on compression strength. Chemosphere, v.77, p.1152-1156, 2009.

20. FARIA, K. C. P.; GURGEL, R. F.; HOLANDA, J. N. F. Recycling of sugarcane bagasse ash waste in the production of clay bricks. Journal of Environmental Management, v.101, p.7-12, 2012.

21. FERREIRA, G.C., DAITX, E.C. Características e especificações da areia industrial. Geociências, v.19, n.2, p.235-242, 2000.

22. FRÍAS, M.; VILLAR, E.; SAVASTANO, H. Brazilian sugar cane bagasse ashes from the cogeneration industry as active pozzolans for cement manufacture. Cement \& Concrete Composites, V.33, p.490-496, 2011.

23. GONZÁLEZ-LÓPEZ, J. R.; RAMOS-LARA,J. F.; ZALDIVARCADENA, A.; CHÁVEZ-GUERRERO, L. MAGALLANES-RIVERA, R. X.; BURCIAGA-DÍAZ O. Small addition effect of agave biomass ashes in cement mortars. Fuel Processing Technology, v.133, p.35-42, 2015.

24. IBRAHIM, A. R. B.; ROY, M. H.; AHMED, Z. U.; IMTIAZ, G. Analyzing the dynamics of the global construction industry: past, present and future. Benchmarking: an International Journal, v.17, p.232-252, 2010.

25. INSTITUTO BRASILEIRO DE MINERAÇÃO - IBRAM. Informações e Análise da Economia Mineral Brasileira. 2012. Disponível em: <http://www.ibram.org.br/sites/1300/1382/00002806.pdf >. Acesso em 8 de dezembro de 2015. 